\title{
Synergistic antitumor effect of JAWSII dendritic cells and interleukin 12 in a melanoma mouse model
}

\author{
ŁUKASZ ZAPAŁA, RAFAŁ WOLNY, MAŁGORZATA WACHOWSKA, MAREK JAKÓBISIAK and WITOLD LASEK
}

Department of Immunology, Centre of Biostructure Research, Medical University of Warsaw, 02-097 Warsaw, Poland

Received September 5, 2012; Accepted November 16, 2012

DOI: $10.3892 /$ or.2012.2193

\begin{abstract}
One of the possible ways to augment dendritic cell (DC) efficacy in presentation of tumor antigens to effector $\mathrm{T}$ cells is pulsing them with tumor-cell lysates and incubation with certain immunostimulators. We present the results of an immunotherapeutic approach in a murine B78-H1 model using as a vaccine JAWSII DCs in combination with IL-12. Prior to the in vivo experiments, phenotypic characterization of JAWSII cells was performed and optimal conditions for stimulation of these cells were established. As no production of IL-12 by JAWSII cells was found, injections of this cytokine were introduced to vaccination protocols. Three vaccination schedules have been tested: i) prophylactic, ii) therapeutic-intratumoral, and iii) therapeutic-systemic. In all the protocols, vaccination with pulsed + stimulated JAWSII cells in combination with IL-12 was superior to the treatment with either agent alone and led to eradication of the tumor in several cases. The results of the study may be helpful in planning optimal DC-based therapeutic protocols in cancer patients.
\end{abstract}

\section{Introduction}

Dendritic cells (DCs) are the most potent antigen-presenting cells, critical for the induction of specific immune responses $(1,2)$. Despite some differences between the murine and human immune system, DC-based cellular vaccines in mouse tumor models may provide significant information on how to optimize therapeutic antitumor approaches in humans (3). DCs have been proved effective as both prophylactic and therapeutic vaccines in a number of experimental models (4). One of the possible ways to augment DC efficacy in presentation of tumor antigens to effector cells is pulsing them with tumor cell lysates (5). However, DCs pulsed with tumor antigens are not usually able to reach final maturity, which affects their properties to induce antitumor response. Prior to in vivo application, incubation of DCs with certain

Correspondence to: Professor Witold Lasek, Department of Immunology, Centre of Biostructure Research, Medical University of Warsaw, Banacha 1a, 02-097 Warsaw, Poland

E-mail:witold.lasek@wum.edu.pl

Key words: dendritic cell, interleukin 12, melanoma, cancer vaccine immunostimulators is therefore necessary. The change in their phenotype greatly enhances their antigen-presenting properties $(6,7)$. It appears that DCs stimulated in such a manner are able to further differentiate to respond to factors secreted by the host immune system.

As the immune response of the host may be suppressed by the tumor, injections of adjuvants/cytokines (such as IL-12) in combination with cellular vaccines may have a crucial effect on overcoming tumor evasion mechanisms $(8,9)$. Systemic injections of IL-12 lead to enhancement of natural killer (NK) and cytotoxic T-cell activity and result in the increase of IFN- $\gamma$ production, as well as in affecting differentiation of antigenspecific Th lymphocytes $(10,11)$. IL-12 was found to effectively inhibit the growth of several experimental tumors in mice, via IFN- $\gamma$ and T cell-dependent pathways, as well as due to antiangiogenic effects $(12,13)$.

JAWSII cells, immortalized C57BL/6 murine bone marrow-derived DCs, seem to be a promising candidate for use in cancer therapy in a cellular vaccine approach $(14,15)$. In a previous study, we analyzed phenotype and functional properties of JAWSII cells and optimized conditions of their stimulation (16). The purpose of this study was to determine the in vivo applicability of JAWSII cells as a component of DC-based cellular vaccine used in combination with IL-12 in a murine B78-H1 melanoma model. Three antitumor approaches of this combination have been tested: prophylactic, local (intratumoral), and systemic therapy.

\section{Materials and methods}

Cells. JAWSII cells were purchased from the American Type Culture Collection (CRL-11904). The cells were grown in RPMI-1640 medium (Sigma-Aldrich, St. Louis, MO, USA) supplemented with 10\% non-inactivated FCS (GibcoInvitrogen, Paisley, Scotland, UK), antibiotics (penicillin + streptomycin + amphotericin, Sigma-Aldrich), and $5 \mathrm{ng} / \mathrm{ml}$ murine GM-CSF (PeproTech, London, UK). JAWSII cells were maintained in a humidified atmosphere at $37^{\circ} \mathrm{C}$ and $5 \%$ $\mathrm{CO}_{2}$ and passaged twice a week.

B78-H1 cells, an amelanotic clone of the murine melanoma B16 cell line, were primarily provided by Dr L.H. Graf (Chicago, IL, USA) and were found to be suitable in our previous immunotherapeutic models $(17,18)$. The cells were cultured in Dulbecco's modified Eagle's medium (DMEM, Sigma-Aldrich) supplemented with $10 \%$ inactivated FCS and 
antibiotics. Other culturing conditions were similar to those used for JAWSII cells.

For in vivo experiments, B78-H1 cells were trypsinized, washed twice in ice-cold PBS and resuspended in PBS at the concentration of $2 \times 10^{5} / 20 \mu 1$ per mice (or in some experiments $5 \times 10^{5} / 20 \mu$ l per mice). Cell viability was determined prior to the inoculation using trypan blue exclusion test and estimated to be $>90-95 \%$.

In vitro JAWSII stimulatory conditions. In the present study, we used a protocol of stimulation of JAWSII cells that was previously described (16). In brief, JAWSII cells were pulsed with tumor cell lysates (JAWSII cells to B78-H1 melanoma cells ratio $=1: 1$ ) for $3 \mathrm{~h}$ followed by a 48 -h incubation with polyriboinosinic polyribocytidylic acid (poly I:C sodium salt, Sigma-Aldrich, concentration in cultures, $100 \mu \mathrm{g} / \mathrm{ml}$ ) and interferon $\gamma$ (recombinant mouse IFN- $\gamma$, BD Pharmingen ${ }^{\mathrm{TM}}$, $10 \mathrm{ng} / \mathrm{ml}$ in cultures).

Mice. For in vivo experiments, C57BL/6 mice (8-week-old, bred in a local animal facility and kept in conventional conditions) were used. All the experiments were approved by the Local Ethics Committee.

Flow cytometric analysis of stimulated JAWSII cell phenotype. The following monoclonal antibodies, previously described (16), were used to study surface markers of JAWSII cells: anti-MHC class I, anti-MHC class II, anti-CD11c, anti-CD40, anti-CD80, anti-CD86, anti-CCR7, and anti-CD8 $\alpha$; relevant isotype controls were used. Anti-CD80 and anti-CD86 double staining was performed so as to determine simultaneous expression of both CD80 and CD86. Surface markers were analyzed after pulsing JAWSII cells with tumor-cell lysates and incubation with appropriate immunostimulators at concentrations described above. The cells were then collected, resuspended in PBS with $0.5 \%$ BSA and $0.05 \%$ sodium azide ( 0.5 million cells in $50 \mu \mathrm{l})$, incubated for $30 \mathrm{~min}$ at $4^{\circ} \mathrm{C}$ with appropriate $\mathrm{mAbs}$, and analyzed in FACS Scan (BectonDickinson). The level of surface marker expression, estimated by the mean fluorescence intensity (MFI), was analyzed using CellQuest software.

Phagocytosis assay. JAWSII cells were stained with $1 \mathrm{mM}$ DiI for $10 \mathrm{~min}$ at $37^{\circ} \mathrm{C}$ and washed two times with ice-cold PBS and seeded in 12-well plates. The following day, JAWSII cells were stimulated with $10 \mathrm{ng} / \mathrm{ml} \mathrm{IFN}-\gamma$ or/and $100 \mu \mathrm{g} / \mathrm{ml}$ poly I:C. Twenty-four hours later, B78-H1 melanoma cells were stained with $5 \mu \mathrm{M} \mathrm{CFSE}$ for $10 \mathrm{~min}$ at $37^{\circ} \mathrm{C}$, washed two times with ice-cold PBS, and placed in a $25 \mathrm{~cm}^{2}$ culture flask. After 24 h, B78-H1 cells were lysed by the freeze/thaw method, added in a volume of $100 \mu \mathrm{l}$ and cocultured with JAWSII cells for an additional $3 \mathrm{~h}$ at $37^{\circ} \mathrm{C}$ and $5 \% \mathrm{CO}_{2}$ or at $4^{\circ} \mathrm{C}$. Then, the cells were collected, washed and resuspended in $300 \mu 1$ of PBS. A total of 10,000 cells were analyzed on a FACS Scan (Becton-Dickinson) using CellQuest Pro Software Version 5.2. Phagocytosis level was measured as a percentage of double positive cells.

$I L-12$. Recombinant mouse IL-12 (specific activity $4.6 / 10^{6} \mathrm{U} / \mathrm{mg}$ protein) was a generous gift from the Genetics Institute
CD11c

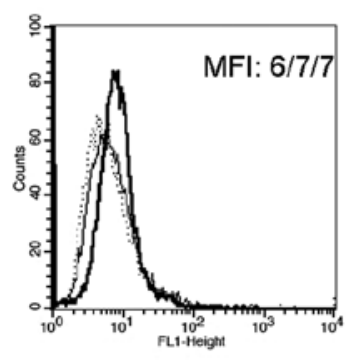

\section{CD80}

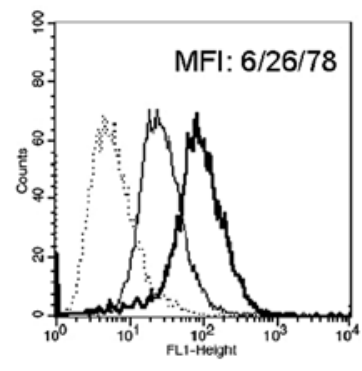

CD8 $\alpha$

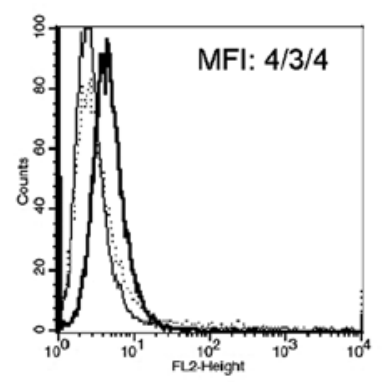

MHC class I

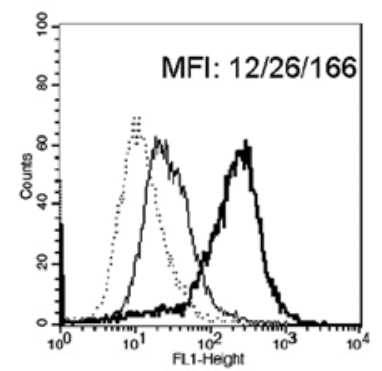

CD40

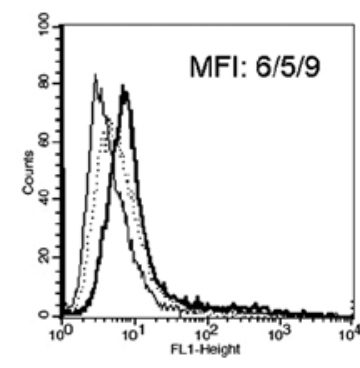

CD86

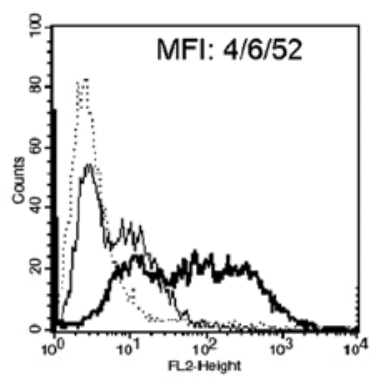

CCR7

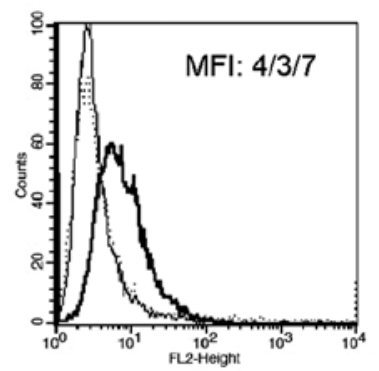

MHC class II

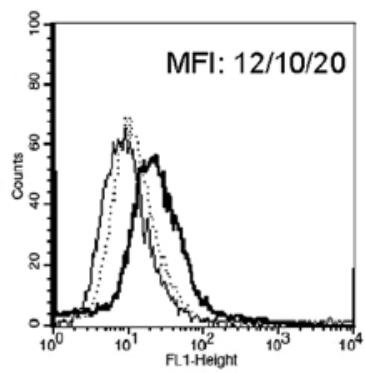

Figure 1. Expression of CD11c, CD40, CD80, CD86, CD8 $\alpha$, CCR7, and MHC class I and II molecules on JAWSII cells pulsed with tumor cell lysates (B78-H1) and then incubated with poly I:C $(100 \mu \mathrm{g} / \mathrm{ml})$ and IFN- $\gamma(10 \mathrm{ng} / \mathrm{ml})$. Thick lines represent specific staining of stimulated cells. Relevant isotype controls are shown as dotted lines and specific staining of unstimulated cells is presented as thin lines. JAWSII cells were pulsed with tumor cell lysates and then incubated with both immunostimulators, followed by washing and immunostaining as described in Materials and methods. MFI = mean fluorescence intensity of isotype control/unstimulated JAWSII cells/stimulated JAWSII cells.

(Cambridge, MA, USA) (18). For in vivo experiments, the cytokine was diluted with $0.1 \%$ BSA (Sigma Chemicals, St. Louis, MO, USA). 


\section{$\mathrm{A}-37^{\circ} \mathrm{C}$}
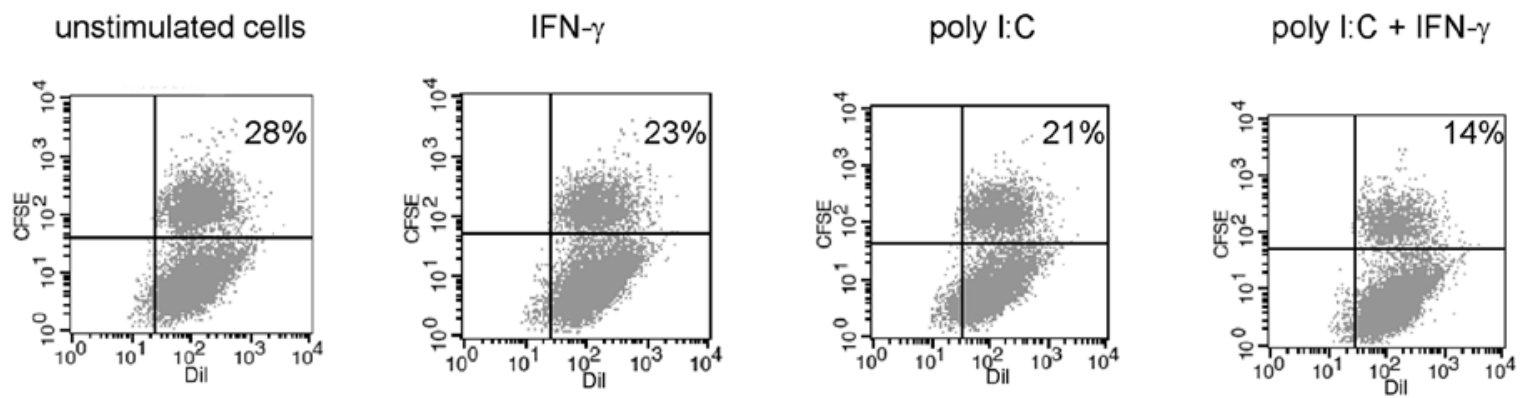

\section{$\mathrm{B}-4^{\circ} \mathrm{C}$}

unstimulated cells

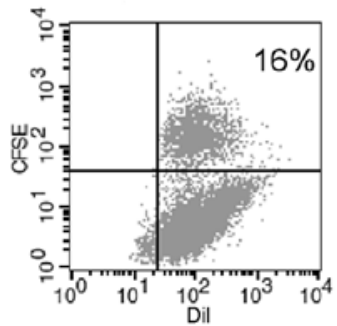

IFN- $\gamma$

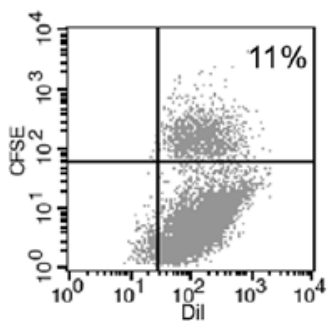

poly I:C

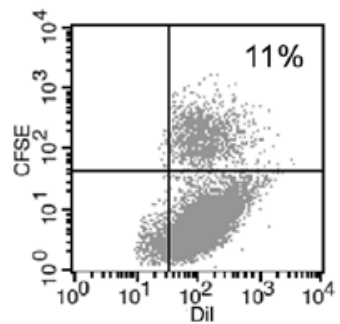

poly I:C + IFN- $\gamma$

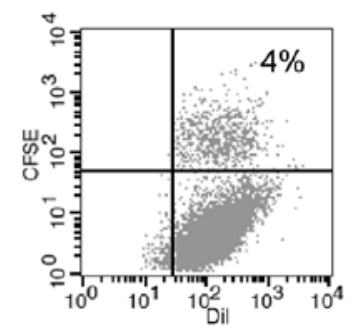

Figure 2. Phagocytosis of frozen-thawed B78-H1 melanoma cells by JAWSII cells at $37^{\circ} \mathrm{C}(\mathrm{A})$ and at $4{ }^{\circ} \mathrm{C}(\mathrm{B})$. The JAWSII cells were stained with $1 \mathrm{mM}$ DiI. For the following $48 \mathrm{~h}$, JAWSII cells were stimulated with poly I:C $(100 \mu \mathrm{g} / \mathrm{ml})$ and IFN- $\gamma(10 \mathrm{ng} / \mathrm{ml})$ alone or in combination. Twenty-four hours after staining the JAWSII cells, tumor B78 cells were stained with $5 \mu \mathrm{M}$ CFSE and cultured for the next $24 \mathrm{~h}$. Then, frozen-thawed B78-H1 melanoma cells were added to the JAWSII cultures and incubated for $3 \mathrm{~h}$ prior to FACS analysis. The JAWSII cells that phagocytosed lysed B78-H1 cells (double positive cells) are presented in the upper right quadrant (percentage).

In vivo protocols used in the B78-H1 melanoma model. Three variants of experiments with JAWSII cells were performed: a) prophylactic, b) therapeutic - intratumoral, and c) therapeutic - systemic schemes.

In the prophylactic scheme, randomly selected mice were injected into the footpad of the left hind limb with stimulated JAWSII on Day -7. On days: $-6,-5$ and -4 mice were administered with IL-12 or diluent (0.1\% BSA in PBS). On Day 0, all the mice were inoculated with B78-H1 melanoma cells in the right hind limb.

In the intratumoral therapeutic scheme, all the mice were inoculated with B78-H1 melanoma cells into the footpad of the right hind limb. Next, on Day +3 , mice were injected into the same site with stimulated JAWSII cells. On Days $+4,+5$ and +6 , mice were injected with IL-12 or diluent (0.1\% BSA in PBS).

In the systemic therapeutic scheme, mice were inoculated with B78-H1 melanoma cells into the footpad of the right hind limb. Next, on Day +3 , mice were injected into the contralateral hind limb with stimulated JAWSII or PBS. On Days: $+4,+5$, and +6 mice were treated with IL-12 or diluent (0.1\% BSA in PBS).

Statistical analysis. Results of experiments on FACS are presented on histograms together with MFI or percentage values. Statistical analysis of the in vivo experiments was performed with the Mann-Whitney-Wilcoxon test (tumor diameter in $\mathrm{mm}$ measured seven weeks after tumor cell inoculation) and the log-rank test (percentage of mice without tumor) using Statistica ${ }^{\circledR}$ software. For clarity, 3 levels of statis- tical significance were used: $\mathrm{p}<0.05 ; \mathrm{p}<0.01$ and $\mathrm{p}<0.001$ that were related to the control group (unless otherwise indicated).

\section{Results}

Expression of surface markers in the stimulated JAWSII cells. Prior to the in vivo experiments in a murine melanoma model, JAWSII cells were pulsed with B78-H1 lysates followed by $48 \mathrm{~h}$ of incubation with poly I:C + IFN- $\gamma$. Then, the influence of this treatment on the expression of surface markers of the cells was determined. In some experiments, FACS analysis of the stimulated JAWSII cells that were additionally incubated with IL-12 for the following $24 \mathrm{~h}$ was performed. As presented in Fig. 1, the cells pulsed with tumor cell lysates and incubated with poly I:C + IFN- $\gamma$ were characterized by high expression of CD80, CD86, and MHC class I molecules. Moreover, there was a moderate increase in the expression of CD40, CCR7, and MHC class II molecules in the cells.

A supplementary incubation with IL-12 (for $24 \mathrm{~h}$ ), after pulsing with tumor lysates and a 48-h incubation with poly I:C + IFN- $\gamma$, did not result in a change of surface markers when compared to the cells analyzed without IL-12 (data not shown).

Phagocytosis of the frozen-thawed B78-H1 melanoma cells by JAWSII cells. The ability of JAWSII cells to phagocytose B78-H1 lysates was determined in the experiment on FACS with CFSE dye. It was found that JAWSII cells phagocytosed frozen-thawed B78-H1 melanoma cells effectively. As 
A

$$
\begin{aligned}
& \text { - JAWS } \|+ \text { IL-12 } \\
& \text { - - - JAWSII } \\
& \text {-.- IL-12 } \\
& \text { — control group }
\end{aligned}
$$

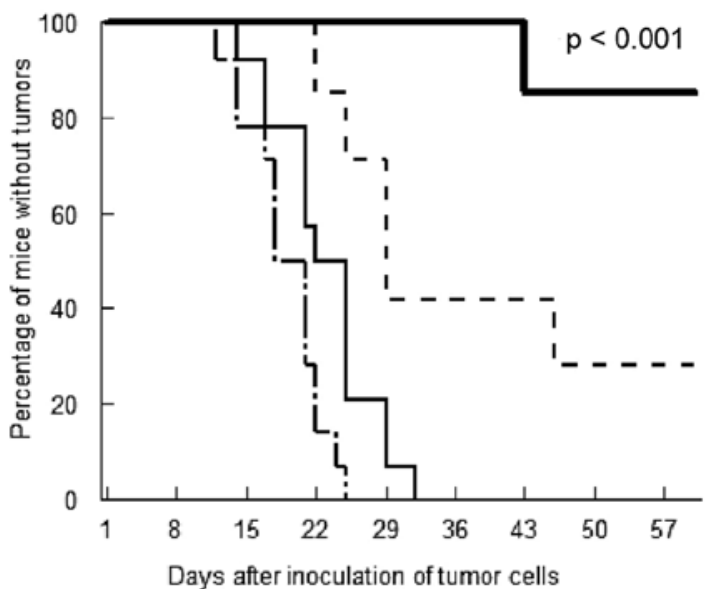

B

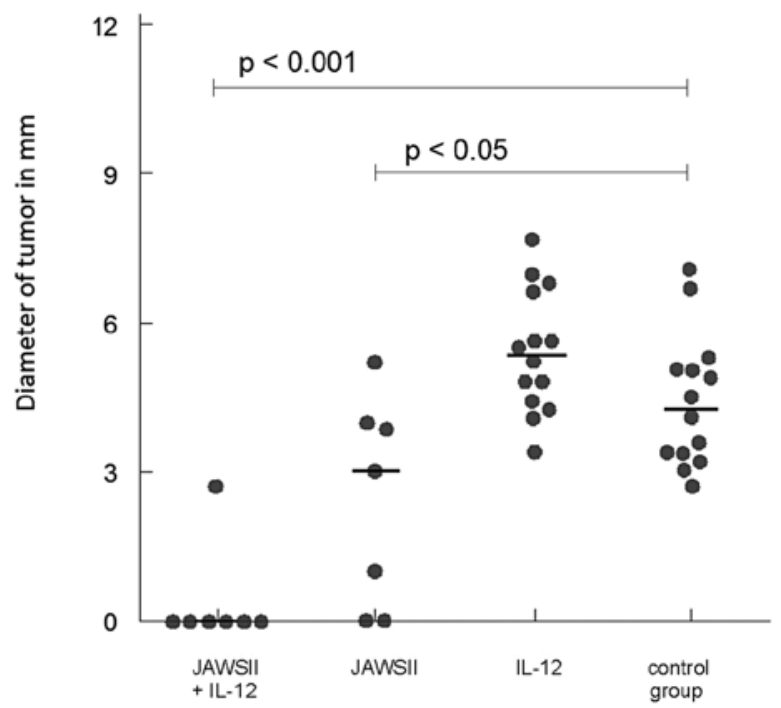

Figure 3. Effects of administration of JAWSII cells and/or IL-12 on the development of B78-H1 melanoma - prophylactic scheme. JAWSII cells (pulsed with B78-H1 cell lysates and incubated with poly I:C and IFN- $\gamma$ as described in Materials and methods) were injected into the footpad of the left hind limb on Day -7 , followed by injection of IL-12 on Days -6, -5 and -4. On Day 0, tumor cells were inoculated into the contralateral limb. P denotes probability vs. control. (A) Percentage of mice without tumor. (B) Tumor diameter in mm measured seven weeks after tumor-cell inoculation. Horizontal lines denote medians.

A

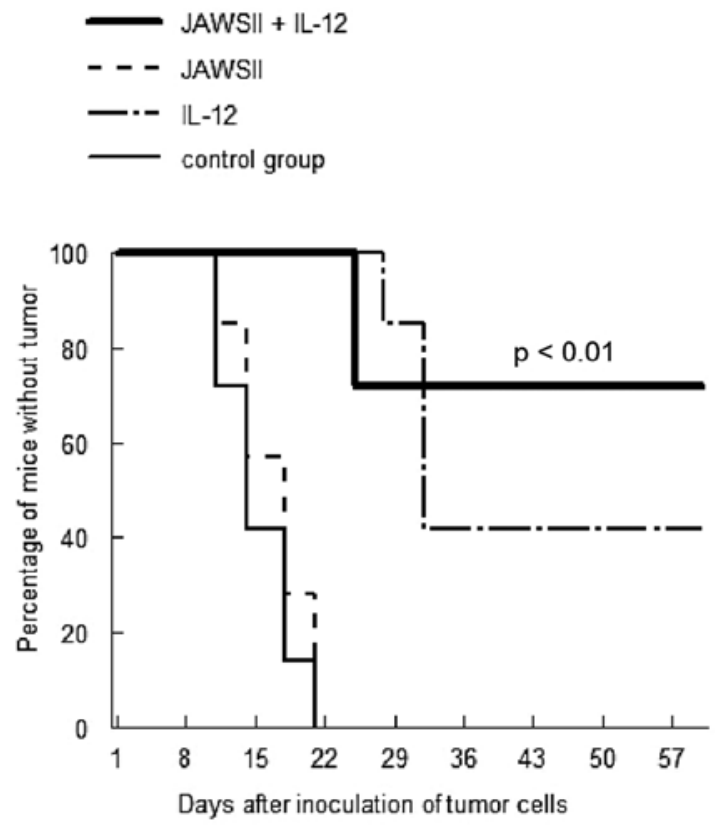

B

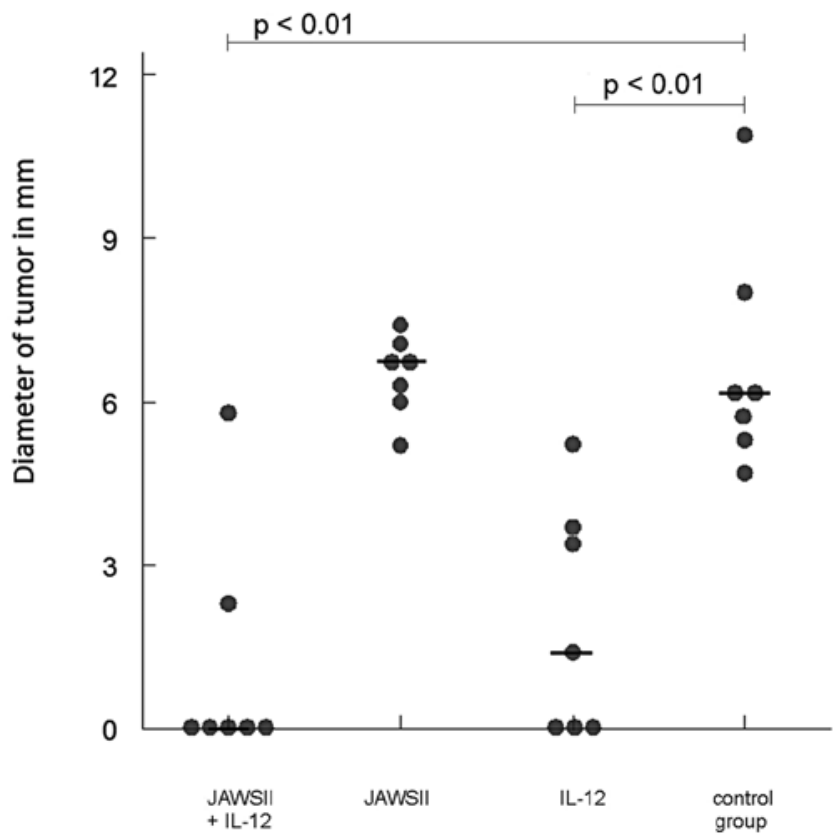

Figure 4. Effects of administration of JAWSII cells and/or IL-12 on the development of B78-H1 melanoma - therapeutic intratumoral scheme. JAWSII cells (pulsed with B78-H1 cell lysates and incubated with poly I:C and IFN- $\gamma$ as described in Materials and methods) were injected on Day +3 into the same footpad of the hind limb in which tumor cells were inoculated (Day 0), followed by injection of IL-12 on Days $+4,+5$ and +6 . P denotes probability vs. control. (A) Percentage of mice without tumor. (B) Tumor diameter in mm measured seven weeks after tumor-cell inoculation. Horizontal lines denote medians.

presented in Fig. 2, the phagocytic capacity of JAWSII cells was decreased after stimulation with poly I:C $(100 \mu \mathrm{g} / \mathrm{ml})$ and IFN- $\gamma(10 \mathrm{ng} / \mathrm{ml})$, particularly in combination of these two agents.
Production and secretion of IL-12 by JAWSII cells. IL-12 was not present in supernatants from unstimulated JAWSII cultures and JAWSII lysates, using an ELISA kit (Mouse IL-12p70 ELISA MAX ${ }^{\mathrm{TM}}$ Set Deluxe, Biolegend, Inc., San Diego, CA, 
A
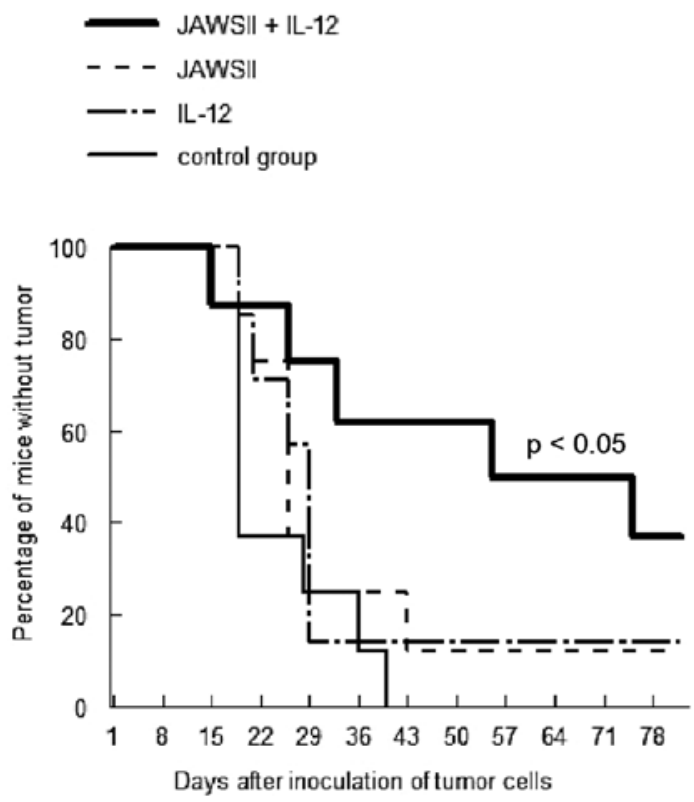

B

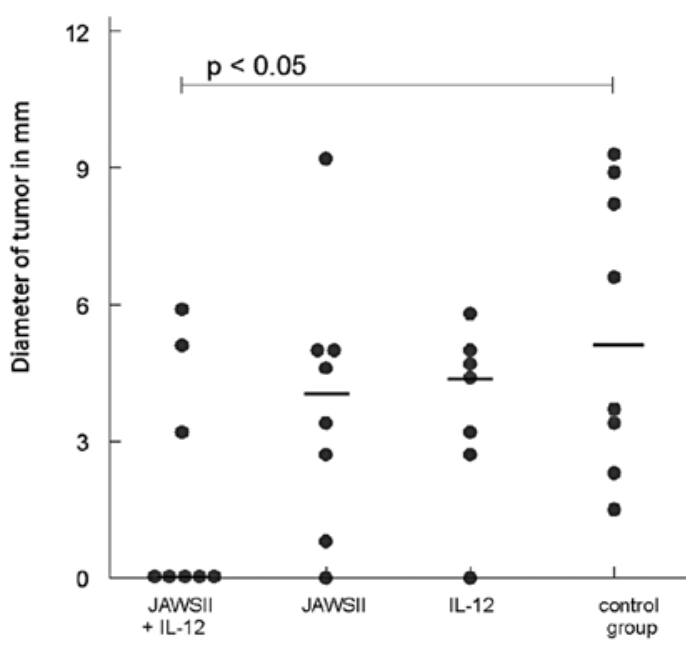

Figure 5. Effects of administration of JAWSII cells and/or IL-12 on the development of B78-H1 melanoma - therapeutic systemic scheme. Following tumor-cell injection into the footpad of the right hind limb, JAWSII cells (pulsed with B78-H1 cell lysates and incubated with poly I:C and IFN- $\gamma$ as described in Materials and methods) were injected into the contralateral hind limb on Day +3 , followed by injection of IL- 12 on Days $+4,+5$ and +6 . P denotes probability vs. control. (A), Percentage of mice without tumor. (B), Tumor diameter in mm measured seven weeks after tumor-cell inoculation. Horizontal lines denote medians.

USA) (data not shown). It was also revealed that neither poly I:C nor IFN- $\gamma$, alone or in combination, stimulated secretion of IL-12 in 24-h JAWSII cultures.

\section{In vivo experiments}

Prophylactic scheme. As shown in Fig. 3, injection of JAWSII cells pulsed with tumor cell lysates and incubated with poly I:C + IFN- $\gamma$ followed by the administration of IL-12 induced effective immunization; no tumor was observed in $86 \%$ of mice at the end of the experiment $(\mathrm{p}<0.001)$. Injection with JAWSII cells alone resulted in some preventive effect; no tumor development in $29 \%$ of cases ( $p<0.01$ vs. control), which was statistically inferior when compared with the group treated with JAWSII + IL-12 (p<0.05). Furthermore, smaller diameters of tumors were observed in that group ( $\mathrm{p}<0.05 \mathrm{vs}$. control). No prophylactic effect was demonstrated in the group of mice treated with IL-12 alone.

Intratumoral therapeutic scheme. In the experiment, combination of JAWSII cells pulsed with B78-H1 cell lysates + incubated with poly I:C + IFN- $\gamma$ and IL-12, when injected intratumorally, led to tumor eradication in $71 \%$ of cases $(p<0.01 \mathrm{vs}$. control) and significantly smaller diameters of tumors at the end of the observation period (median $=0$, $\mathrm{p}<0.01$ vs. control) (Fig. 4). On the contrary, in the group of mice treated with JAWSII cells alone, all the mice developed neoplasms. Injection with IL-12 alone led to some curative effect (eradication of tumors in $43 \%$ of mice) $(p<0.01$ vs. control) and a decrease of tumor diameter $(\mathrm{p}<0.01$ vs. control). However, no statistical difference was found between the group treated with JAWSII cells + IL-12 and the group injected with IL-12 alone, with regard to the rate of tumor development and diameter of tumors.
Systemic therapeutic scheme. Results of the experiment on the efficacy of systemic antitumor therapy consisting of stimulated JAWSII \pm IL-12 are presented in Fig. 5. The combination of JAWSII cells and IL-12 resulted in a significant rate of tumor eradication; $38 \%$ of mice did not develop tumors at the end of observation period ( $\mathrm{p}<0.05$ vs. control), and smaller diameters of tumors were observed 7 weeks after tumor cell inoculation $(p<0.05$ vs. control). Injections with either JAWSII cells or IL-12 alone did not lead to a significant therapeutic effect; no statistically significant differences were noted in comparison with the control group.

\section{Discussion}

Among different methods of introduction of antigenic material into DCs (tumor lysates, peptides, RNA, DNA), the use of lysates of whole tumor cells is the most frequently described protocol $(5,19,20)$. However, it was claimed that DCs pulsed with tumor antigens are unable to reach maturity and produce insufficient amounts of IL-12 to induce efficacious antitumor response (7). As JAWSII cells did not produce IL-12, neither unstimulated nor stimulated (poly I:C, IFN- $\gamma$ alone and in combination), we decided to introduce injections of IL-12 to in vivo protocols in order to improve the activity of cellular vaccine by modification of tumor environment and eliciting additional antitumoral defense mechanisms. It was found that JAWSII cells pulsed with tumor lysates and incubated with the combination of immunostimulators (poly I:C + IFN- $\gamma$ ) revealed high expression of CD80 and CD86 molecules, and also increased levels of CCR7, and MHC molecules when compared with the control group (Fig. 1). Both in the previous (16) and in the present study, we found that incubation of JAWSII cells with poly I:C and IFN- $\gamma$ increases expression 
of CD40, although some authors argue against the presence of the CD40 molecule in this cell line $(21,22)$. Markedly, further incubation of the cells with IL-12 did not affect the expression of the studied surface markers. In the study of Rossowska et al, pulsing bone marrow-derived DCs with tumor cell lysates did not alter the levels of the surface markers (6). However, a slight decrease in expression of CD86, MHC class I and II molecules was observed when compared with untreated cells (6). In our previous study (16), the decreased ability of endocytosis of JAWSII cells (reflecting more mature stage of these cells) in cultures incubated with LPS or poly I:C was described, while in the present study we demonstrated maturation-inducing potential of combination of poly I:C and IFN- $\gamma$. JAWSII cells are able to phagocytose lysed B78-H1 melanoma cells efficiently and the combination of immunostimulators diminishes that ability (Fig. 2). The impact of appropriate immunostimulators, mostly microbial elements, is crucial for the ability of DCs to promote anticancer response in vivo $(6,7)$. They induce changes in the phenotype of DCs, which start to express high levels of costimulatory and MHC molecules (6). Furthermore, stimulated in that manner, semi-mature DCs retain properties for further maturation in vivo after the contact with the factors secreted by the host's immune system, mainly cytokines. As the immune response is usually suppressed by the tumor, coadministration of IL-12 with the cellular vaccine may induce stronger antitumor mechanisms (9).

In the prophylactic scheme of therapy in our murine melanoma model, JAWSII cells pulsed with tumor lysates and incubated with poly I:C + IFN- $\gamma$ demonstrated significant antitumor effect, which was augmented in the group of mice treated additionally with IL-12 (Fig. 3). It should be noted that the cytokine alone was found to be ineffective. By contrast, in the intratumoral therapeutic scheme, injections of stimulated JAWSII cells alone showed no antitumor effects while administration of IL-12 induced eradication of tumors in some mice (Fig. 4). The best treatment option was, as in the prophylactic protocol, application of JAWSII cells in combination with IL-12; most mice were cured. Despite induction of similar antitumor effects, it is possible that different mechanisms operated in the prophylactic versus the intratumoral therapeutic protocol. As we have shown in our previous studies, injection of activated and tumor lysate-fed JAWSII cells was associated with specific induction of effector cytotoxic $\mathrm{T}$ lymphocytes in regional lymph nodes (16). It is quite probable that supplementation of IL-12 enhanced this specific response in the prophylactic scheme $(9,23)$. In the intratumoral therapeutic schedule, non-specific, NK cell-dependent mechanisms were able to contribute to the overall antitumor effect in the combination treatment, since IL-12 is the strong activator of these cells $(24,25)$. The antiangiogenic effect of IL-12 may also be helpful in the eradication of tumors $(26,27)$. Activation of both specific and non-specific mechanisms of immunity in the combination therapy (JAWSII cell vaccine + IL-12) was strong enough to induce, as shown in Fig. 5, antitumor responses in the most challenging systemic therapeutic scheme, mimicking clinical situation.

IL-12 was used in immunotherapy as a single agent in a number of models of murine cancers (28). However, it should be stressed that although some authors described a decrease in the rate of tumor growth after injection with IL-12, superior effects were noted when the cytokine was used in combination with other forms of therapy $(12,29,30)$. IL-12 may improve stimulating properties of bone marrow-derived DCs in vitro (31). In the study of Tatsumi et al, the use of IL-12 in combination with DCs pulsed with tumor lysates was more effective in comparison with DCs alone in a murine liver cancer model, leading to an induction of specific antitumor responses (8). Furthermore, Fallarino et al observed specific response of cytotoxic lymphocytes after administration of IL-12 and pulsed DCs in nearly $100 \%$ of mice (9). In one clinical study, peripheral blood mononuclear cells (PBMCs) loaded with tumor antigenic peptides and IL-12 induced $\mathrm{CD}^{+}$ T-cell response (23). However, methodological differences (using PBMCs in selected HLA-A2 ${ }^{+}$patients, lack of IL-12 alone-treated controls) makes comparison of this study with our model unreliable.

In conclusion, in the present study we presented antitumor effects of the JAWSII cell-based cellular vaccine used in combination with IL-12 in a murine model of B78-H1 melanoma. The findings of this study may aid in the planning optimal DC-based therapeutic protocols in cancer patients.

\section{Acknowledgements}

This study was supported by the Medical University of Warsaw, Poland (grant nos. 1M19/W2, 1M19/NM5/07) and by the Ministry of Science and Higher Education, Poland (grant no. N N401 011536).

\section{References}

1. Ueno H, Klechevsky E, Morita R, et al: Dendritic cell subsets in health and disease. Immunol Rev 219: 118-142, 2007.

2. Palucka K, Ueno H, Roberts L, Fay J and Banchereau J: Dendritic cell subsets as vectors and targets for improved cancer therapy. Curr Top Microbiol Immunol 344: 173-192, 2012.

3. Gilboa E: DC-based cancer vaccines. J Clin Invest 117: 1195-1203, 2007.

4. Pajtasz-Piasecka E and Indrova M: Dendritic cell-based vaccines for the therapy of experimental tumors. Immunotherapy 2: 257-268, 2010.

5. Palucka K, Ueno $\mathrm{H}$ and Banchereau J: Recent developments in cancer vaccines. J Immunol 186: 1325-1331, 2011.

6. Rossowska J, Pajtasz-Piasecka E, Szyda A, Krawczenko A, Zietara $\mathrm{N}$ and Dus D: Tumour antigen-loaded mouse dendritic cells maturing in the presence of inflammatory cytokines are potent activators of immune response in vitro but not in vivo. Oncol Rep 21: 1539-1549, 2009.

7. Vegh $\mathrm{Z}$ and Mazumder A: Generation of tumor cell lysateloaded dendritic cells preprogrammed for IL-12 production and augmented $\mathrm{T}$ cell response. Cancer Immunol Immunother 52: 67-79, 2003.

8. Tatsumi T, Takehara T, Kanto T, et al: Administration of interleukin-12 enhances the therapeutic efficacy of dendritic cell-based tumor vaccines in mouse hepatocellular carcinoma. Cancer Res 61: 7563-7567, 2001.

9. Fallarino F, Uyttenhove C, Boon T and Gajewski TF: Improved efficacy of dendritic cell vaccines and successful immunization with tumor antigen peptide-pulsed peripheral blood mononuclear cells by coadministration of recombinant murine interleukin-12. Int J Cancer 80: 324-333, 1999.

10. Gately MK, Warrier RR, Honasoge S, et al: Administration of recombinant IL-12 to normal mice enhances cytolytic lymphocyte activity and induces production of IFN-gamma in vivo. Int Immunol 6: 157-167, 1994.

11. McKnight AJ, Zimmer GJ, Fogelman I, Wolf SF and Abbas AK: Effects of IL-12 on helper T cell-dependent immune responses in vivo. J Immunol 152: 2172-2179, 1994. 
12. Brunda MJ, Luistro L, Rumennik L, et al: Antitumor activity of interleukin 12 in preclinical models. Cancer Chemother Pharmacol 38 (Suppl): S16-S21, 1996.

13. Nastala CL, Edington HD, McKinney TG, et al: Recombinant IL-12 administration induces tumor regression in association with IFN-gamma production. J Immunol 153: 1697-1706, 1994.

14. Pajtasz-Piasecka E, Rossowska J, Szyda A, Krawczenko A and Dus D: Generation of anti-tumor response by JAWS II mouse dendritic cells transduced with murine interleukin 12 genes. Oncol Rep 17: 1249-1257, 2007.

15. Xu Y, Darcy PK and Kershaw MH: Tumor-specific dendritic cells generated by genetic redirection of Toll-like receptor signaling against the tumor-associated antigen, erbB2. Cancer Gene Ther 14: 773-780, 2007.

16. Zapala L, Drela N, Bil J, Nowis D, Basak GW and Lasek W: Optimization of activation requirements of immature mouse dendritic JAWSII cells for in vivo application. Oncol Rep 25: 831-840, 2011.

17. Basak GW, Zapala L, Wysocki PJ, Mackiewicz A, Jakobisiak M and Lasek W: Interleukin 15 augments antitumor activity of cytokine gene-modified melanoma cell vaccines in a murine model. Oncol Rep 19: 1173-1179, 2008.

18. Switaj T, Jalili A, Jakubowska AB, et al: CpG immunostimulatory oligodeoxynucleotide 1826 enhances antitumor effect of interleukin 12 gene-modified tumor vaccine in a melanoma model in mice. Clin Cancer Res 10: 4165-4175, 2004.

19. Ballestrero A, Boy D, Moran E, Cirmena G, Brossart P and Nencioni A: Immunotherapy with dendritic cells for cancer. Adv Drug Deliv Rev 60: 173-183, 2008.

20. Palucka $\mathrm{K}$ and Banchereau J: Cancer immunotherapy via dendritic cells. Nat Rev Cancer 12: 265-277, 2012.

21. Haase C, Michelsen BK and Jorgensen TN: CD40 is necessary for activation of naive $\mathrm{T}$ cells by a dendritic cell line in vivo but not in vitro. Scand J Immunol 59: 237-245, 2004.

22. Jorgensen TN, Haase $\mathrm{C}$ and Michelsen BK: Treatment of an immortalized APC cell line with both cytokines and LPS ensures effective T-cell activation in vitro. Scand J Immunol 56: 492-503, 2002.
23. Peterson AC, Harlin $\mathrm{H}$ and Gajewski TF: Immunization with Melan-A peptide-pulsed peripheral blood mononuclear cells plus recombinant human interleukin-12 induces clinical activity and T-cell responses in advanced melanoma. J Clin Oncol 21: 2342-2348, 2003.

24. Wehner R, Dietze K, Bachmann M and Schmitz M: The bidirectional crosstalk between human dendritic cells and natural killer cells. J Innate Immun 3: 258-263, 2011.

25. Manetti R, Parronchi P, Giudizi MG, et al: Natural killer cell stimulatory factor [interleukin 12 (IL-12)] induces T helper type 1 (Th1)-specific immune responses and inhibits the development of IL-4-producing Th cells. J Exp Med 177: 1199-1204, 1993.

26. Bielawska-Pohl A, Blesson S, Benlalam $\mathrm{H}$, et al: The anti-angiogenic activity of IL-12 is increased in iNOS ${ }^{-1-}$ mice and involves NK cells. J Mol Med 88: 775-784, 2010.

27. Airoldi I, Di Carlo E, Cocco C, et al: Endogenous IL-12 triggers an antiangiogenic program in melanoma cells. Proc Natl Acad Sci USA 104: 3996-4001, 2007.

28. Hill HC, Conway TF Jr, Sabel MS, et al: Cancer immunotherapy with interleukin 12 and granulocyte-macrophage colony-stimulating factor-encapsulated microspheres: coinduction of innate and adaptive antitumor immunity and cure of disseminated disease. Cancer Res 62: 7254-7263, 2002.

29. Kozar K, Kaminski R, Switaj T, et al: Interleukin 12-based immunotherapy improves the antitumor effectiveness of a low-dose 5-Aza-2'-deoxycitidine treatment in L1210 leukemia and B16F10 melanoma models in mice. Clin Cancer Res 9: 3124-3133, 2003

30. Grohmann U, Bianchi R, Ayroldi E, et al: A tumor-associated and self antigen peptide presented by dendritic cells may induce $\mathrm{T}$ cell anergy in vivo, but IL-12 can prevent or revert the anergic state. J Immunol 158: 3593-3602, 1997.

31. Kelleher P and Knight SC: IL-12 increases CD80 expression and the stimulatory capacity of bone marrow-derived dendritic cells. Int Immunol 10: 749-755, 1998. 